\title{
Self-Calibrating Body Sensor Network Based on Periodic Human Movements
}

\author{
Haider A. Sabti, Student Member, IEEE \& David V. Thiel, Senior Member, IEEE \\ Griffith School of Engineering, Griffith University \\ Brisbane, QLD Australia \\ h.al-husseinawi@griffith.edu.au,d.thiel@griffith.edu.au
}

\begin{abstract}
Body sensor networks with a central gateway node can control wireless transmission and optimize the network lifetime. The central node should be placed within communication reach of other nodes spread out around the human body, such as on the chest. This paper reports a novel energy-efficient time multiplexing transmission method based on the human rhythmic movement of running for on-body wireless communication. The running style of each individual allows the network to selfcalibrate the communication scheme so that transmissions occur only when high link reliability is predicted. This technique takes advantage of the periodic running actions to implement a dynamic time division multiple access (TDMA) strategy for a five node body network with very little communication overhead, long sleep times for the sensor transceivers and robustness to communication errors. The results showed all wireless communications were successful, except when two nodes attempt to use the transmission medium simultaneously. An aggregated network reliability of $90 \%$ was achieved compared to $63 \%$ when employing traditional time multiplexing algorithms.
\end{abstract}

Keywords-WBAN; WSN; TDMA; Wireless Communication; Smart Sensors; Sports engineering; Running; Gait Running Cycle

\section{INTRODUCTION}

$\mathrm{H}$ EALTH AND SPORT applications have captured the attention of researchers to develop sensing technologies and to adopt wireless communication systems instead of traditional wired connections. Wearable/implanted wireless body area networks (WBAN) not only offer mobility for users, but also provide real time feedback by continuously monitoring and analyzing sensed information. This can help professionals to effectively measure the athlete training sessions and perform early diagnosis and treatment. Many studies have been carried out to provide patients with the best and most comfortable care possible, to monitor athletes performance closely in sport fields and for assisted living and entertainment [1-4].

Unlike regular wireless sensor networks (WSN), there are specific challenges associated with human body monitoring. The most important difference is the need for reliable communication with each WBAN node, as opposed to the redundant character of information sensing and exchange using in existing WSN nodes. A comprehensive comparison between the differences of WSN and WBAN can be found in [5].
Network reliability is defined by the speed and amount of information received. This is an important part of any wireless network and can be affected by many parameters including the number of nodes, overlapping transmissions and total amount of traffic generated on the network [6]. Many wireless communication techniques have been adopted to increase the network capacity and enhance the reliability such as CSMA/CA (carrier sense multiple access with collision avoidance), TDMA (time division multiple access), FDMA (frequency division multiple access) and CDMA (code division multiple access). However, these approaches are subject to energy cost, such as the idle listening phase in the case of CSMA; time synchronization required by standard TDMA which requires additional energy to send and receive timing information between all network sensors to get their clocks synchronized. Furthermore, FTDM and CMDA require complex hardware to perform frequency hopping and frequency signal spreading techniques [7].

This paper introduced a human gesture transmission method based on the dynamic time division mapping. The technique allows the sensed acceleration data to trigger information broadcast at specific actions, so that the energy cost for multiple transmissions can be completely avoided and the lifetime of the network can be prolonged.

\section{PREVIOUS WORK}

The promising features of BSN have encouraged healthcare providers and sport sponsors to invest more in all aspects of these networks. In [8] a study was made on different runners with different skills to investigate the kinematic changes resulting from fatigue. Another study [9] introduced passive synchronization for network sensors using the heartbeat rhythm instead of the traditional periodic beacons, thus network time synchronization is achieved without the need to use a radio transceiver.

The authors previous work was carried out to determine the best node locations on a human body and to achieve a maximum connectivity to a receiving unit located on the chest, in order to sustain good wireless communications between the nodes while the athletes are moving [10-13]. In [10] a wireless accelerometer sensor module was used to determine the link performance by recording the data and traffic loss on different runners and for different transmitter locations around the human body (foot, leg and arm). An approximate swing time 
calculation algorithm was employed to find the swing time effect on these losses. This study showed the effect of different runners, sensor location and data rate on the link performance. Also the auto acknowledgment feature of the transceiver chip was explored and assessed for different applications.

Recent measurements were reported on the channel occupancy and traffic generated to provide high reliability for a time multiplexed-star shape body network [12]. Results showed a network reliability of $95 \%$ achieved by retransmitting the lost packets. The drawback is the reduced network life time as more power is consumed compared to its normal operation.

\section{SYSTEM AND MEASUREMENT SETTINGS}

\section{A. Wireless sensor node design}

The network involved the use of five wireless transceiver sensor nodes which were developed at Griffith university [14]. The nodes were modified to include the wireless transceivers. Each node consisted of a MCU (ATmega324P Microcontroller), wireless transceiver chip module (NRF24L01+) with a built-in PCB meander-line monopole antenna, accelerometer sensor (MMA7260QT) with acceleration Sensitivity of (2g), memory (AT45DB161D) and a power source (shown in Fig. 1 (a)) in the form of $3.6 \mathrm{~V}$ battery with a maximum capacity of $240 \mathrm{~mA}$. The battery capacity gives a node lifetime of around three hours, which is sufficient to monitor a complete training session. The nodes were programmed to work as transceivers to allow two way communications and were able to store transmitted data and/or received from other nodes. The codes that drive the node operations were implemented using $\mathrm{C}$ language and Atmel Studio 6.1.

The network uses a hub node positioned on the chest which communicates with the four nodes located on the wrists and thighs as shown in Fig. 1 (b). The received data at the hub node can be identified by the unique node transmission address. The recorded data can be downloaded to a personal computer using the UART interface (CP2102) at each node which allows wired data transfers for real-time monitoring and further processing during or after the completion of the sport activity.

The tests involved the participation of three human volunteers and were conducted under the project code for responsible conduct of research (Ethics approval ENG/20/13/HREC). The participants were asked to wear the wireless transceiver nodes using fabric bands attached to their arms and legs to ensure stability during running and for easy on-body mounting. All measurements were conducted in an outdoor environment to minimize possible multipath effects from nearby objects. The nodes were configured to transmit and receive data at the same power level, data rate and frequency $(0 \mathrm{dBm}, 2.4 \mathrm{GHz}$ and $2 \mathrm{Mbps})$.

\section{B. Acceleration Axis Selection and Data Resolution}

The three-axis accelerometer sensor measures the analog acceleration data in three orthogonal directions. Any movement

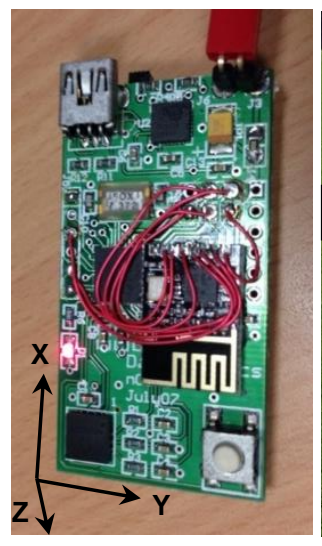

(a)

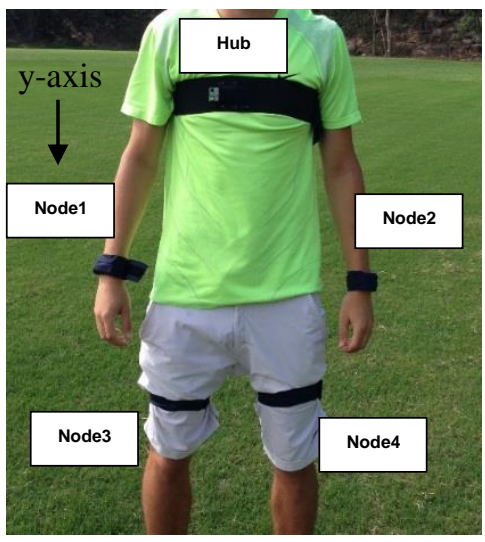

(b)
Fig. 1. (a) Hardware structure of the wireless sensor node (b) Position of wireless nodes on the human body.

of the sensor at any given direction will produce an analog voltage reading at the sensor output which is converted by the 10 digit $\mathrm{MCU}$ analogue to digital convertor (ADC) to a digital form of $(\mathrm{mV} / \mathrm{g})$. The hardware limitation of MCU results in a range of digital values between $(0-1023)$ which is the 10th power of 2 (1024). The highest voltage resulted from accelerations of $(2 \mathrm{~g})$ is converted to a digital value of 1023 .

The three axes give three different readings depending on the movement of the body. Choosing the best readings to detect movement gesture or action is dependent on the direction in which the sensor is moved. The data are usually recorded in the memory and transmitted or received using the wireless transceiver. In all of the experiments conducted in this paper, the sensors were placed in a horizontal position at the wrists and thighs (the y-axis is vertical to the ground when the person is standing) as shown in Fig. 1. In one test, a human volunteer was asked to run a small distance of $20 \mathrm{~m}$ and the acceleration data were recorded for the three axes at the four positions to find most suitable acceleration readings at each sensor location. The test showed that in running, the y-axis for all four positions gives more detailed and clear readings than the other axes, and so it was used in the following tests to calculate the swing time (two consecutive foot contacts with the ground).

Another important feature is the acceleration data resolution or the sampling rate. The sampling rate is programmable and can be set differently for different applications. A low sample rate of $(10 \mathrm{~Hz})$ would give 10 acceleration points in one second for the human movement and therefore it will not be enough to identify gestures for fast activities (running as an example). A larger sample rate (such as $200 \mathrm{~Hz}$ ) will give more details than required (200 sample per second) and consequently will consume more power from both the transmitter and receiver as well as the channel capacity and memory. Therefore, $100 \mathrm{~Hz}$ sampling rate was used as an optimized choice to fit the needs of this study [10]. 


\section{EXPERIMENTAL SETUP AND RESULTS}

This section describes the operational steps of three algorithms that were employed in the conducted tests using the system shown in Fig. 1. All nodes were configured to record their acceleration data and to transmit it to the hub node whenever they receive a data request from the hub. As shown in Fig. 2, the hub sends an acceleration sample request to each node at a given time burst, and immediately switches to the receive mode and saves the received data. The transmission address is changed to communicate with the next node. The communication sequence of the hub is repeated starting at Node 1 and ending at Node 4. The reason for the two way communication (represented by the hub communication request) is that each node has its own clock system and if they were programmed to transmit at different times, eventually their clocks will drift apart. This will result in timing inaccuracy and even data loss. The transceiver capability of each node allows it to transmit data and switch back to the receiving mode for reception within the defined sampling rate. With each sample request sent by the hub node, a counter is incremented (location counter) and logged in the hub memory. This counter helps identify the sample number of each acceleration value. The locations at which samples were lost for each node can be identified. In all trials the sample rate was set to $100 \mathrm{~Hz}$.

\section{A. Standard Time Division Multiplexing Algorithm}

1) Algorithm Description: Before carrying outdoor experiments the network was tested indoors to validate the standard time multiplexing algorithm. The hub was set to transmit 1000 requests for each node with a $10 \mathrm{~ms}$ timing burst. This provides a test time of 10 s (resulted from multiplying the 1000 samples by $10 \mathrm{~ms}$ timing burst) which is more than the time required for following running tests. As expected, the test shows no sample losses or any shift in the hub timing sequence. Fig. 3 shows the sequence in which acceleration samples were received and saved in the hub memory along with its sample number and node number for 10 consecutive samples. It shows Node 1 data always logged first and so on.

The $10 \mathrm{~ms}$ timing burst was selected based on timing measurements. This included the total time of data on air (ToA) for both transmitting and receiving operations, sensor switching time from transmitting to receiving modes and the timing requirements for the microcontroller communications process with the transceiver chip, memory and accelerometer sensor. If the hub is communicating with only one node, this will result in 100 samples per second for that node. However, since the hub in the network is controlling four nodes then each node will provide 25 samples in one second (the accumulated number of samples for the four nodes is 100). Therefore, the calculated time difference between the samples will be $40 \mathrm{~ms}$ for each node, given that the swing time in running for normal humans is around $400 \mathrm{~ms}$. The result will be 10 samples in a swing which is not sufficient to capture fast movement. A simple solution is given by logging the acceleration from only two nodes (Node $1 \&$ Node 3 ) or (Node $2 \&$ Node 4 ). In running these two nodes move oppositely to each other alternating the

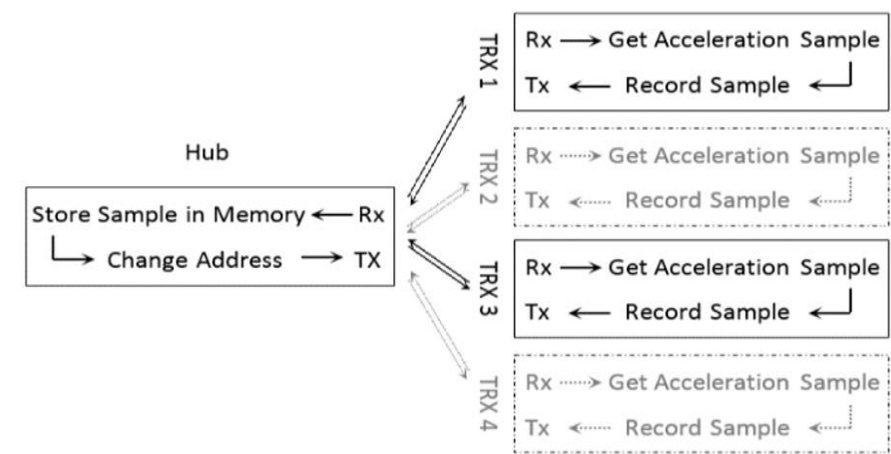

Fig. 2. Shows the network standard timing multiplexing between the hub and other nodes.

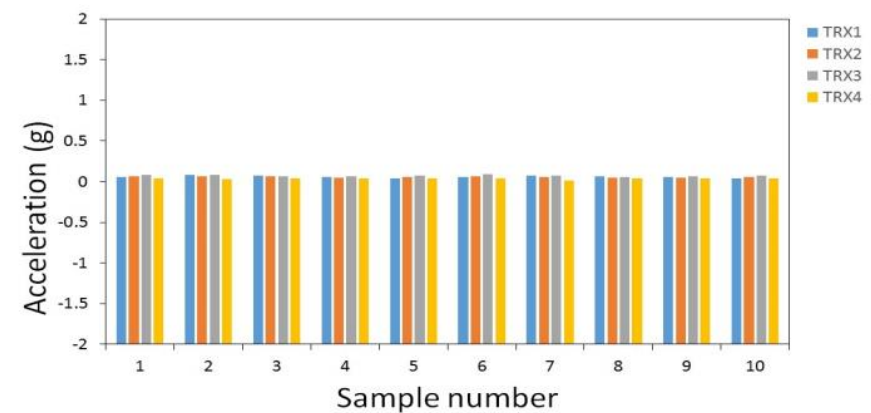

Fig. 3. The hub order sequence of received acceleration samples for Node 1, 2 , 3 and 4 in laboratory. These are the transmissions received over a total of $0.4 \mathrm{~s}$.

line of site with the chest. Since running is a periodic movement, it can be assumed that the acceleration data for the first pair of nodes are the mirror of the second pair. This technique will results in a double number of samples (50 samples per second per node) for the $100 \mathrm{~Hz}$ sampling rate network, and will provide more details for the acceleration pattern and will help in gesture identification.

In the outdoor test, participant A was asked to run for a small distance $(20 \mathrm{~m})$ wearing all four nodes. The standard time division algorithm operated for only opposite nodes, and the distance is sufficient for reaching constant speed. The participant details and the average successful data transmission rate during one running cycle are shown in Table I. Fig. 4 shows the acceleration samples recorded and lost (missed) along with the location counter for Node 1 and Node 3 . The acceleration values received by the hub draw a pattern for locations of reliable data communication as shown in Fig 4 (a) and (b). For the wrist locations, the samples are delivered to the hub when the y-axis acceleration is high especially around a maximum value of $2 \mathrm{~g}$ and data is lost when acceleration values are dropping. On the contrary for the thigh locations, samples are delivered to the hub when the y-axis acceleration is low especially around a minimum value of $-0.7 \mathrm{~g}$ and data is lost when acceleration value are increasing. These patterns show the high reliability location points for successful communication with the hub node for the wrist and thigh during running. The empty space between acceleration points represent the location at which data were lost in either direction (data request from the hub was not received or the acceleration sample form the node was not delivered). 
TABLE I

Participant pool and average successful data transmission rate during one running cycle (for wrist position).

\begin{tabular}{cccccc}
\hline \hline $\begin{array}{c}\text { Participant } \\
\text { ID }\end{array}$ & $\begin{array}{c}\text { Age } \\
\text { (years) }\end{array}$ & $\begin{array}{c}\text { Height } \\
(\mathrm{m})\end{array}$ & $\begin{array}{c}\text { Weight } \\
(\mathrm{kg})\end{array}$ & $\begin{array}{c}\text { Average } \\
\text { Cycle } \\
\text { Time } \\
(\mathrm{ms})\end{array}$ & $\begin{array}{c}\text { Link } \\
\text { availabi } \\
\text { lity / } \\
\text { Cycle }\end{array}$ \\
\hline A & 28 & 170 & 70 & 460 & $62 \%$ \\
B & 32 & 172 & 88 & 440 & $64 \%$ \\
C & 27 & 175 & 90 & 500 & $61 \%$ \\
\hline \hline
\end{tabular}

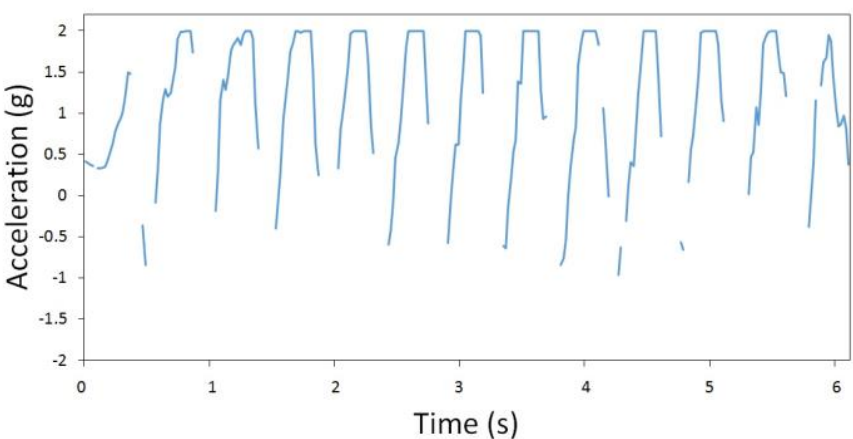

(a)

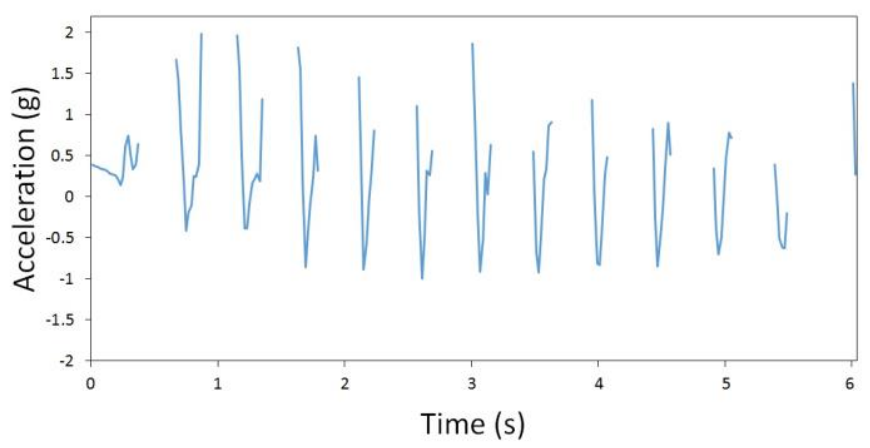

(b)

Fig. 4. The recorded acceleration data $(\mathrm{mV} / \mathrm{g})$ at the hub for participant $\mathrm{A}$ of (a) Node 1- right wrist (b) Node 3 - right thigh.

2) Results: The right side of Table I shows the average running cycle time and the average link availability within one running cycle for all three runners for the wrist position. For all participants more than $60 \%$ of the average running cycle there is reliable communication between the hub and the wrist (which means that nearly $40 \%$ of the data is lost when adopting a standard TDM algorithm). It also shows that different running patterns (represented by the wrist cycle time) for the runners affect the wireless link reliability.

In Fig. 5, the acceleration data for the right wrist and thigh (Node 1 and Node 3), left wrist and thigh (Node 2 and Node 4) of participant A were collected and merged in one graph to fully illustrate the body sensor network readings during running. It shows two running cycles for right and left body limbs of in one second, and it can be proved that the cycle for the left/right wrist and thigh are alternating with an average cycle time of around $450 \mathrm{~ms}$ (which is very close to the time measured in Table I). Furthermore, sending the required information at these reliable location points for each node, will save link capacity and ensures data delivery.

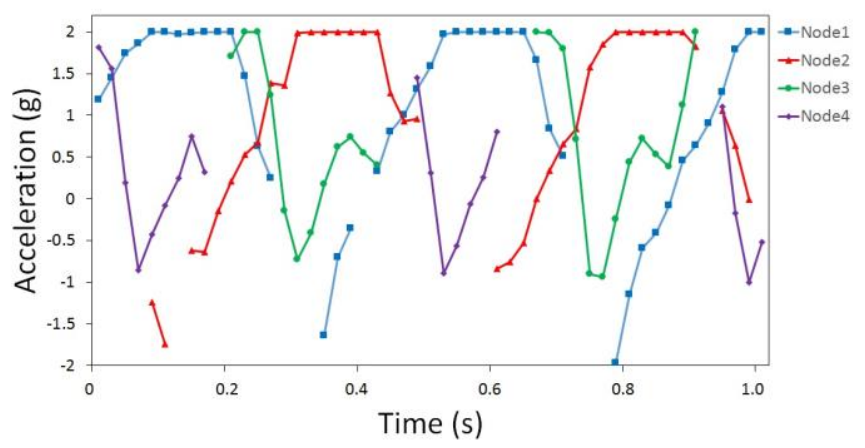

Fig. 5. The received acceleration data $(\mathrm{mV} / \mathrm{g})$ at the hub for all nodes during running.

\section{B. Self Calibrating Algorithm}

1) Algorithm Description: As different runners have different running styles and cycle times (Table I) this would requires different programming for each runner to make sure the data is sent at a specific event. A self-calibrating algorithm was designed to ensure a reliable communication between the hub and the nodes by finding the best transmission points for each node and for different runners. There are two important parameters in determining the best communication time windows based on the acceleration value (actual reading of the sensor) and its location. Controlling one parameter alone would not result in the best performance. For example if the hub node requests data only at specific locations, then it would be unknown if these locations lie within the pattern where consistent communication exist for that specific node (it will act the same as standard TDM only with a bigger time burst). Requesting the nodes to send their data at certain acceleration values will force the nodes to transmit whenever that condition is true and it may result in overlapped and multi-transmission in one running cycle. Thus, a combination of both is required.

Fig. 6 shows more details of the self-calibrating algorithm. The hub node is responsible for performing this algorithm and controlling the time division multiplexing and address changing (Fig. 2). For every 10ms (which is the timing burst) the hub enables the Tx Mode by making a register value (sample=1). This allows it to send a sample data request before switching back to the Rx Mode. After sending the request, the location counter is incremented for every data request for each node (there are four location counters, one for each node). In the Rx Mode, for $10 \mathrm{~ms}$ the hub keeps monitoring the node reply for the request that already been sent, if the time is finished and the hub does not receive anything it will send another request for the next node and so on. For each acceleration sample received and saved in acc[i], the count counter is incremented (there are four count counters, one for each node), and a condition check is applied on the two counters (location and count). If they are not the same and the count counter holds a value more than 10 (this means there are ten or more samples received consequently before the connection was lost) then an arithmetic operation is performed to find the average acceleration value and the average location of these samples. Adopting the middle point is the best way to ensure that transmission occurs reliably. 


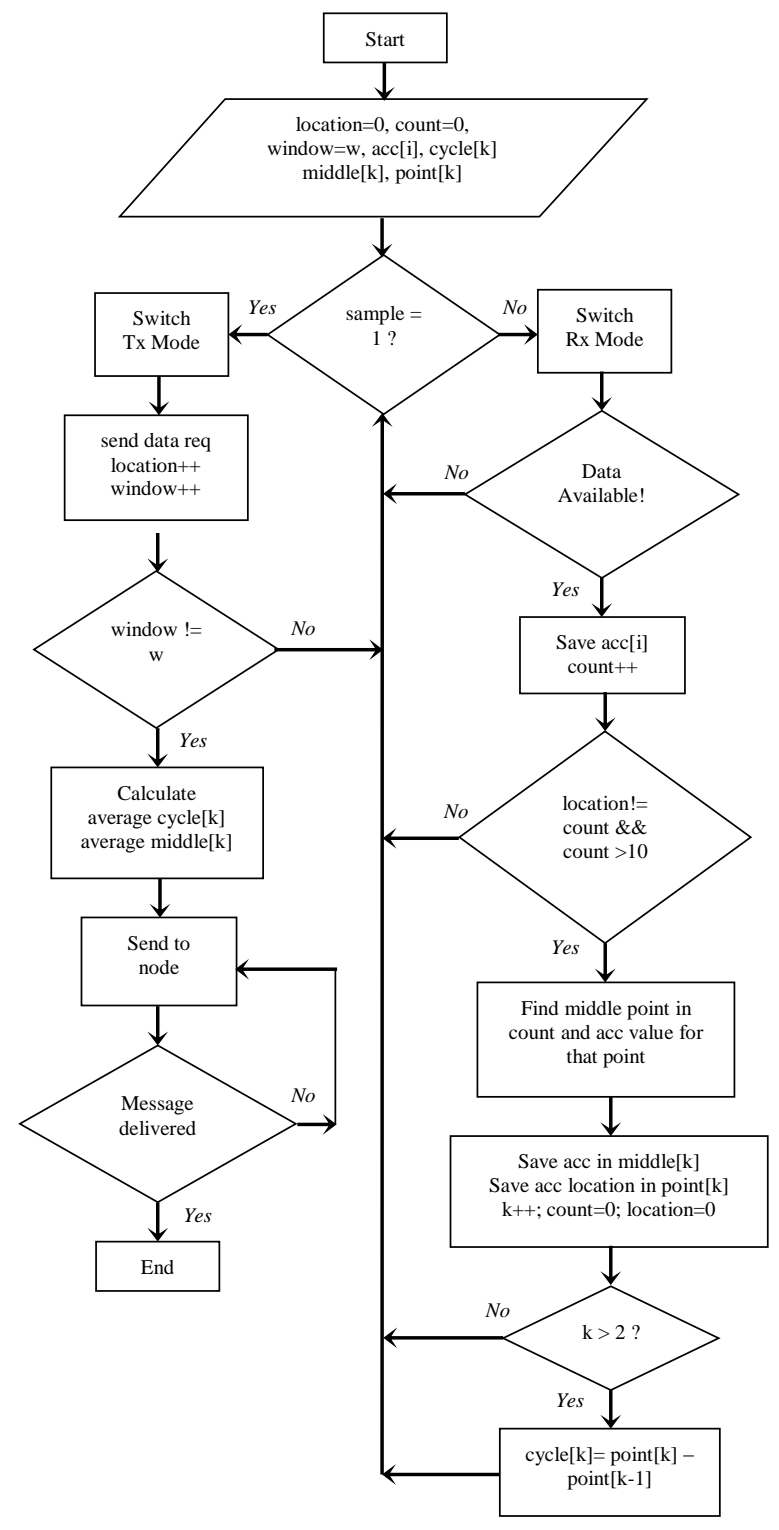

Fig. 6. Flowchart describes the self-learning algorithm performed by the hub. The loop continues for a defined calibration window of samples.

The resultant acceleration value is stored in the middle[k] array, while the acceleration location is saved in the point [k] array. Finding the difference between the average location (point[k-1]) and the next average location calculated from the next 10 or more samples which is obtained through requesting data from the same node (point[k]) will result in finding the running cycle and is saved in cycle $[\mathrm{k}]$. Reaching a predefined counter value of samples (window $=$ w) the hub calculates the average acceleration value and average cycle and sends it (for given attempts to insure delivery) to each node to identify best transmission locations. The nodes depend on these values in their transmission and they follow two conditions to ensure that the data is sent only when the calculated acceleration value is reached and the sample numbers are equal or more than the average cycle length (as shown in Fig. 7).

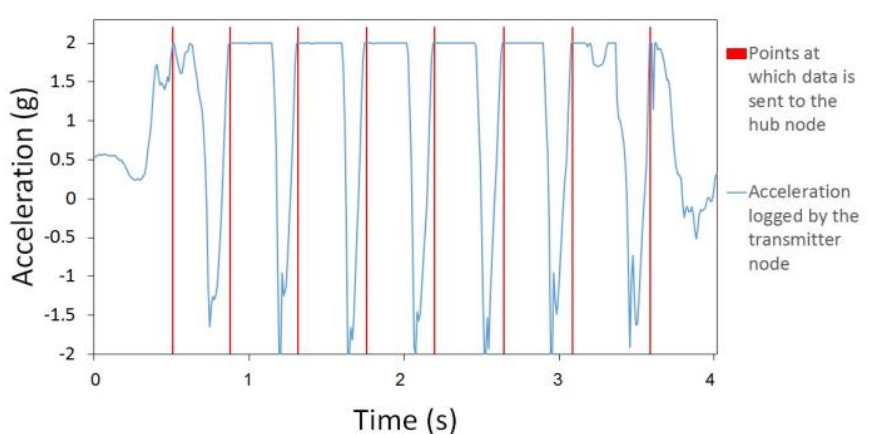

(a)

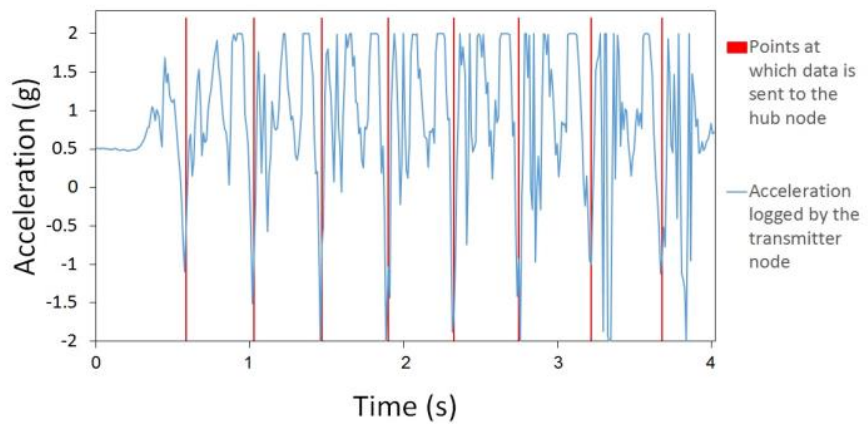

(b)

Fig. 7. Acceleration profile in running showing the location points at which data is received by the hub along with the recorded acceleration samples at each node for (a) wrist (b) thigh.

2) Results: Applying this algorithm to periodic human movements of wrist and thigh during running results in sending one sample per limb cycle. In Fig. 7, one sensor location was tested at a time with the hub. It shows the location points at which data was received by the hub marked in [Red bars] along with the acceleration samples [Blue line] that were saved in the nodes attached to the wrist and thigh of participant $\mathrm{B}$. The nodes send data only at the maximum and minimum acceleration values separated by the running cycle length of participant B $(440 \mathrm{~ms})$ that was obtained in Table I. The figure shows that all communication attempts between the hub and wrist/thigh were successful.

\section{Dynamic Time Division Multiplexing Algorithm}

1) Algorithm Description: In this test, participant B was asked to wear the network shown in Fig. 1 and to run at moderate speed. The nodes were calibrated using the selfcalibrating algorithm performed by the hub initially and all nodes learned to send information at their allocated time slots. Fig. 8 shows the sequence at which the nodes transmit to the hub starting at Node 1 (right wrist), Node 4 (left thigh), Node 2 (left wrist) and then Node 3 (right thigh) which is a typical running movement. Table II shows the transmission attempts for all nodes. This dynamic time division allows nodes to send data depending on the human running gesture, and ensures that only 4 transmissions are allowed in a cycle. Small changes in the limb cycle time would not affect the reliability as the two conditions have to be met before transmission occurs. However, at some point two nodes may meet their condition and attempt to send data at the same time. 


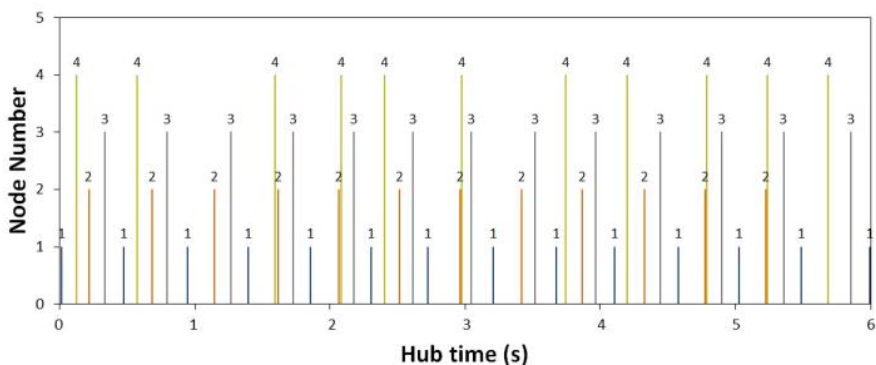

Fig. 8. Timing diagram showing the location points at which data is received by the hub from all four nodes.

TABLE II

Network performance demonstrated by successful wireless transmission using the dynamic time division for all four nodes of participant B.

\begin{tabular}{ccc}
\hline \hline $\begin{array}{c}\text { Node } \\
\text { Number }\end{array}$ & $\begin{array}{c}\text { Average cycle } \\
\text { time for each node } \\
\text { in (ms) }\end{array}$ & $\begin{array}{c}\text { Aggregated link } \\
\text { reliability }\end{array}$ \\
\hline 1 & 450 & $98 \%$ \\
2 & 450 & $86 \%$ \\
3 & 460 & $95 \%$ \\
4 & 470 & $81 \%$ \\
\hline
\end{tabular}

To prevent packet collision, a simple carrier sensing algorithm was adopted at each node to sense the frequency medium for transmission before sending its information. The node checks if a signal is detected and waits for $10 \mathrm{~ms}$ (which is the calculated time burst) and then monitors for a signal again before using the medium. Unsuccessful transmission attempts for Node 4 (near $\mathrm{t}=1 \mathrm{~s}$ ) and Node 2 (near $\mathrm{t}=6 \mathrm{~s}$ ) shown in Fig. 8 can be explained as follows; after the node waiting time is finished the conditions are not true anymore and the nodes have missed their transmission windows. This issue only appeared when there are multiple sensor nodes in the network simultaneously talking to the hub.

2) Results: The results in Table II shows the average limb cycle time (ms) for all nodes attached to participant B for successful transmission only. The limb cycle time is very close with small differences due to the $10 \mathrm{~ms}$ time burst resolution. It also shows an aggregated network reliability of $90 \%$ achieved using dynamic transmission. The $10 \%$ losses are the result of transmission overlap between two nodes leading to an unsuccessful transmission attempt. The table shows that losses resulted from Nodes $1 \& 3$ (right wrist and thigh) are lower than the losses from Nodes $2 \& 4$ (left wrist and thigh). This is because Nodes $4 \& 2$ attempted to communicate with the hub while Nodes $1 \& 3$ were transmitting their data, respectively.

\section{CONCLUSION}

A wireless accelerometer sensor module was used to determine the high reliability locations by recording the data delivered for different node locations (wrists and thighs) on the human body during running. A self-calibrating algorithm was employed to find these time location points and the running cycle of the participants was used in a dynamic time division multiplexing algorithm. The results showed that all transmissions attempts were successful in a node to node communication, and there were a few data losses (10\%) in multi-node network when two nodes were trying to transmit at the same time. Future work will involve the design of an interactive application that presents the data in a more understandable form for the coaches by showing the symmetry between the right and left body limbs during running cycles for different runners.

\section{ACKNOWLEDGEMENT}

This work was supported by the Higher Committee for Education Development in Iraq (HCED) as part of Haider A. Sabti's Ph.D. program. The authors would also like to thank the participants for their participation and Griffith University for granting access to important resources. This research was conducted under Griffith University Ethics approval number ENG/20/13/HREC.

\section{REFERENCES}

11] C. A. Otto, E. Jovanov, and A. Milenkovic, "A WBAN-based system for health monitoring at home," 3rd IEEE/EMBS International Summer School in Medical Devices and Biosensors, pp. 20-23, 2006.

[2] R. Marin-Perianu, M. Marin-Perianu, P. Havinga, S. Taylor, R. Begg, M Palaniswami, and D. Rouffet, "A performance analysis of a wireless body-area network monitoring system for professional cycling," Personal and Ubiquitous Computing, vol. 17, pp. 197-209, 2013.

[3] D. V. Thiel, H. G. Espinosa, G. M. Davis, E. Dylke, N. Foroughi, and S L. Kilbreath, "Arm Movement: The effect of obesity on active lifestyles," Procedia Engineering, vol. 60, pp. 182-187, 2013.

[4] H. G. Espinosa, D. A. James, S. Kelly, and A. Wixted, "Sports monitoring data and video interface using a GUI auto generation Matlab tool," Procedia Engineering, vol. 60, pp. 243-248, 2013.

[5] B. Latré, B. Braem, I. Moerman, C. Blondia, and P. Demeester, "A survey on wireless body area networks," Wireless Networks, vol. 17, pp 1-18, 2011.

[6] J. Zhao and R. Govindan, "Understanding packet delivery performance in dense wireless sensor networks," Embedded networked sensor systems, New York, USA, 2003

[7] S. Marinkovic, C. Spagnol, and E. Popovici, "Energy-efficient TDMAbased MAC protocol for wireless body area networks," Sensor Technologies and Applications, pp. 604-609, 2009.

[8] C. Strohrmann, H. Harms, C. Kappeler-Setz, and G. Troster "Monitoring kinematic changes with fatigue in running using body-worn sensors," IEEE Transactions on Information Technology in Biomedicine, vol. 16, pp. 983-990, 2012.

[9] L. Huaming and T. Jindong, "Heartbeat-driven medium-access contro for body sensor networks," IEEE Transactions on Information Technology in Biomedicine, vol. 14, pp. 44-51, 2010.

[10] H. A. Sabti and D. V. Thiel, "Node position effect on link reliability for body centric wireless network running applications," IEEE Sensors Journal, vol. 14, pp. 2687-2691, 2014.

[11] H. A. Sabti and D. V. Thiel, "A study of wireless communication links on a body centric network during running," Procedia Engineering, vol. 72, pp. 3-8, 2014

[12] H. A. Sabti and D. V. Thiel, "Time multiplexing-star shape body sensor network for sports applications," IEEE APS/URSI, Memphis, 2014.

[13] H. A. Sabti and D. V. Thiel, "Movement based time division multiplexing for near real time feedback body area network applications," IEEE International Workshop on Antenna Technology, Sydney, pp. 23-25, 2014.

[14] D. A. James, N. Davey, and T. Rice, "An accelerometer based sensor platform for insitu elite athlete performance analysis," IEEE Sensors, vol.3, pp. 1373-1376, 2004 


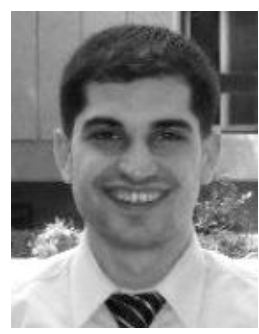

Haider A. Sabti received the B.Sc. degree in electronics and communication engineering from Al-Nahrain University, Baghdad, Iraq, in 2007, and the M.Sc. degree in electronics and communication engineering in 2010. He is currently pursuing his Ph.D. degree in wireless body area networks for running sport applications. $\mathrm{He}$ is an IEEE student member and a staff member of the Griffith School of Engineering in Brisbane, Australia. He worked as an RF engineer in a CDMA communication company in Baghdad, Iraq, since 2010 . He is supported by a scholarship from HCED in Iraq. His current research interests include wearable wireless sensor networks, design and implementation of smart sensors, embedded systems for athletes monitoring, and sport data analysis.

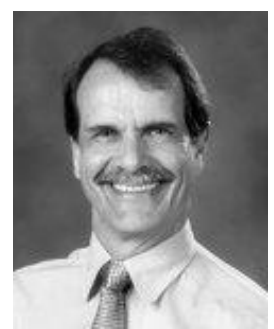

David V. Thiel received a B.Sc. degree in physics and applied mathematics from the University of Adelaide, Adelaide, Australia, and the Masters and Ph.D. degrees from James Cook University, Townsville, Australia. $\mathrm{He}$ is currently Deputy Head of School (Research) in the Griffith School of Engineering, Brisbane, Australia. He has co-authored the book Switched Parasitic Antennas for Cellular Communications. He has published over 150 journal papers, more than 200 papers presented at international conferences, and he has co-authored more than nine patent applications. He was a co-inventor of the new RoHS and WEEE compliant electronics manufacturing technology called "circuits in plastic." His current research interests include electromagnetic geophysics, sensor development, electronics systems design and manufacture, and antenna development for wireless sensor networks. He is a Fellow of the Institution of Engineers, Australia. $\mathrm{He}$ is currently the Chair of the IEEE Wave Propagation Standards Committee and serves on the IEEE Antenna Standards Committee. 